struction. Basic studies are being made of tidal oscillations, with particular attention to conditions in rivers, straits and shallow seas. A start has been made with studies of turbulence associated with the streams. Some experience has been gained in the use of electronic analogues. Statistical studies at the Earthquake Research Institute show that there is a tendency for the monthly mean sea-level to drop sharply before the large earthquakes.

Theoretical work on oceanic circulation presents many difficulties due to lack of information about actual velocities and mixing processes, and to the rapidly increasing complexity of the reasoning as the basic assumptions are made more and more realistic. The output of the Japanese workers in this field, largely owing to the influence of Prof. Hidaka, is enough to set ideas and conclusions running ahead of the observations and experiments. Marine research in Japan is likely to become remarkably productive if the theoretical workers are more actively helped and invigorated by the physicists, engineers and ships. There is a good measure of co-operation, but the practical facilities voted for attacks on obvious practical problems have to be devoted largely to methods which have been proved. This often means that the theoretical workers have to spend ton long with pen and paper before they can put new ideas to work, and have their work stimulated, intensified and improved by practice and closer inquiry. This is a universal problem, but anyone attempting to survey marine science in Japan cannot fail to be impressed by the great wealth of observations, some of which have to be dealt with in fairly general terms, and by a similar wealth of theoretical work, some of which has to seek rather hard for the measurements that it believes most significant. The Japanese scientists lose no opportunity of checking measurements with hydrodynamical theory, and are well aware of the need to devote as much of the practical effort as possible to basic studies; but they might be able to do more if the responsibilities and burden of some of the practical requirements were shared by academic and technical workers in a laboratory in which both were well represented.

Some twenty Japanese scientists are making active contributions to the chemistry of the sea, including the development of new analytical methods and studies of variations in oxygen, carbon dioxide, nitrogen, phosphorus and other nutrients in relation to productivity, water movements and sediments. Attention is being paid to the elements in minor concentrations and to the geochemical implications of the work. Measurements of radioactivity near Bikini atoll have been used for studies of diffusion.

Japan has twenty marine biological stations, each with a staff of two to five full-time research workers as well as technical assistants and facilities for working in coastal waters. They lie between $33^{\circ}$ and $43^{\circ} \mathrm{N}$. over a very wide range of exposure and water conditions. In some the investigations are mainly physiological, without much immediate application to ecological and oceanographic problems. The local faunas are described, though not as completely as for some European laboratories. For work in the open ocean they rely on co-operation from the hydrographic, meteorological and fisheries laboratories, and active work is being done on productivity, indicator species, and the general character of regional faunas in relation to oceanographic factors. They emphasize the need for more taxonomic studies.

The Japanese instrument makers arranged an exhibition in connexion with the Unesco meetings, and it was apparent that they are keeping pace with new developments in marine research.

The International House of Japan was an ideal house for the meetings. The lecture hall will seat two hundred, and the dining hall and adjacent lounge could be combined to accommodate about the same number. There are forty-two bedrooms, single and double, and the service and meals were excellent. It is a non-profit organization, made possible by $£ 100,000$ collected in Japan and a grant of $£ 125,000$ capital expenditure and $£ 25,000$ a year for the first five years from the Rockefeller Founda. tion. It was formally opened in June 1955, and its activities aim primarily at promoting and sponsoring the international exchange and co-operation of intellectual leaders and creative artists, though some of its programmes are planned for students and the general public. The Japanese writer and lawyer Shigeharu Matsumoto is its managing director.

G. E. R. Deacor

\title{
RESISTANCE OF INSECTS TO INSECTICIDES
}

$\mathrm{O}^{\mathrm{N}}$ January 9 the Association of Applied Biologists and the Society of Chemical Industry held a joint symposium in London on the subject of the resistance of insects to insecticides. The morning session $w$. $u s$ devoted to a discussion of the changes in resistance that can occur naturally before any kind of selection has occurred. The afternoon session was chiefly concerned with a discussion of the resistance that may develop following the use of insecticides.

The first paper, by C. Potter, dealt with the variations in resistance to insecticides that normally occur throughout the life-cycle of an insect and of the effect that differences in nutrition may have on resistance. It was pointed out that, in practice, insecticides are usually applied to kill a particular instar of a pest and it is important to choose the most susceptible instar for attack. Furthermore, since there can be variation of resistance with the age of the instar, detailed knowledge of the extent of these changes is desirable if control measures are to be put on a rational basis. Examples were then given to show the extent of the changes in resistance to insecticides that can occur throughout the life-cycle of an insect and also those that can occur during the development of a single instar. The changes throughout the life-cycle may be large; in one example quoted, it was more than 250 times. Smaller changes are found to occur within the instar, but even these can be appreciable, an example of a difference of more than sixteen times being given.

The examples given by Dr. Potter served to show that the extent and direction of the changes in resistance depend on the insecticide used, the species of insect and the conditions of treatment. It follows 
from this that not only may the absolute toxicity of any one insecticide differ with the stage of development, but also that the relative toxicity of any two insecticides is likely to differ also. There appears to be no information on the reasons for the differences in resistance between instars, and they are likely to be complex. Differences in resistance within an instar have been correlated with morphological and physiological changes that occur during its development, but these changes are not necessarily the cause of the differences. Examples were given to prove that the diet of the insect may affect its resistance to insecticide. This was shown to happen both by changing the constitution of artificial diets and by changing the host plants of insects that foed on living plants. The amount of food administered and the period when it was administered were also shown to affect resistance.

A. H. McIntosh then discussed the effect of tem. perature on the toxicity of insecticides. He stated that a change in the temperature at which insects are kept after treatment with poison nearly always affects the kill. If the kill increases with temperature, the temperature coefficient of kill is positive, and vice versa. In contact tests on grain beetles, the size of the temperature coefficient depends on the particle size used if the poison is a solid. If the temperature coefficient is negative, it is always bigger for colloid than for crystals; this happens with DDT and some of its analogues, rotenone and endrin. But if the temperature coefficient is positive, it may be bigger for colloid than for crystals, as with dieldrin, or bigger for crystals than colloid, as with 2-bromomercurithiophen.

The time elapsing between treatment of insects and counts of kill may sometimes affect the size and sign of the temperature coefficient. Thus, the temperature coefficient of dieldrin by injection into mealworms did not change as time passed (one to four days); but with rotenone the coefficient was negative if counts of kill were made up to about a day and a half after treatment, and after this it was positive. At the higher temperature, the percentage kill increased as time passed, but at the lower temperature the insects recovered from the effects of rotenone. The size and sign of the temperature coefficient may also depend on the dose, the method of application, the insect species and the range of temperatures used. The most that can be said about any one poison is that its temperature coefficient is usually positive or negative, as the case may be. Differences in temperature are certainly one cause of differences in both relative and absolute toxicities found by different workers.

K. F. Goodwin-Bailey then read a paper on behalf of $J$. M. Holborn on the susceptibility to insecticides of laboratory cultures of an insect species. The results of tests of the comparative resistance of strains of Tribolium castaneum (the rust-red flour be日tle) and of strains of Calandra granaria (the granary weevil) were described. None of these strains had been exposed previously to insecticides, yet they showed consistent differences in resistance to the test poisons. A laboratory strain of $C$. granaria was found to be between three and $a$ half and six and $a$ half times as susceptible to pyrethrum powders as a wild strain. There was little difference in susceptibility when pyrethrins synergized with piperonyl butoxide were used. Several strains of $T$. castaneum developed from cultures from different laboratories were tested against $\gamma-\mathrm{BHC}$, the pyrethrins and the pyrethrins synergized with piperonyl butoxide. The strain showed marked differences in susceptibility to $\gamma-\mathrm{BHC}$ and the pyrethrins, but the differences were less marked with the synergized pyrethrins.

The most resistant strain was four times as resistant to $\gamma$-BHC and three times as resistant to the pyrethrins as the least resistant strain. There is evidence that these natural differences were inherited through at least six generations. The differences in susceptibility found are thought to be due to differ. ences in vigour. The results also show that the factor of synergism can differ greatly with the different strains. With the species examined, the synergist appears to be most effective against the most resistant strains, thus tending to diminish the differences found with the pyrethrins alone. The data presented in this paper provide evidence that considerable differences in resistance to insecticides can exist between strains of the same species, before any treatment with a poison has taken place; that is, that insecticide resistance is not always acquired by selection.

The first paper in the afternoon was read by J.R. Busvine, who discussed the occurrence and status of insecticide-resistant strains. He listed twenty-one species recorded since 1940 as having insecticideresistant strains in the field. Only those records were listed which at the same time were sub. stantiated with good supporting evidence for the existence of insecticide resistance. The criteria to be adopted in judging whether or not a resistant strain had been produced in the field were then discussed, and it was pointed out that the factors of natural high resistance, faulty insecticide or inefficient application, the elimination of parasites and predators and natural fluctuations in the insect popula. tion, should first be excluded. These factors make the field assessment of resistance difficult, and it should therefore be confirmed in the laboratory by a quantitative comparison with insects of the same species that have not been exposed to an insecticide.

The necessity for adequate standardization of the method of assessment of resistance was stressed, since errors can occur due to lack of standardization of the insect material, and different results may be given by varying the method of test. In general, high resistance is associated with flat probit/regression lines. The probit lines for resistant strains are nearly always flatter than for normal strains. Where two techniques of measurement are used, one may show a bigger difference between resistant and normal than the other ; it generally occurs that the technique showing the bigger difference has the flatter probit line.

Dr. Busvine then considered the types of resistance that occur and concluded that the more important forms of resistance which result in the failure of control measures are characteristically specific to one type of insecticide. The grouping of the insecticides into types on this basis may indicate that they have a similar mode of action. The biochemical basis of resistance has been studied by some workers, and it has been shown that resistant strains have an increased capacity to detoxify DDT, although this alone is not sufficient to account for the degree of resistance that eccurs. It was pointed out that there is some evidence of increased metabolism of the insecticide with strains resistant to $\gamma$-BHC, chlordane and toxaphene. Studies on the genetical bases of resistance have yielded very conflicting results, and there appears to be no general agreement. Dr. Busvine ended his talk with a plea for further research on this subject. 
The next speaker, F. R. Bradbury, then gave a paper on the absorption and metabolism of benzene hexachloride in normal and resistant houseflies. The pick-up and absorption of vapours of $\gamma-\mathrm{BHC}$ and of its non-toxic $\alpha$-, $\beta$ - and $\delta$-isomers by the housefly Musca domestica have been studied by confining susceptible and resistant strains over treated filter papers; labelling with carbon-14 and chlorine-36 has facilitated analysis. The general picture of resistance to $\gamma$-BHC deduced from the data broadly parallels that currently held for DDT. Resistant flies pick up and absorb less poison and perform a more rapid conversion to excretable metabolites with both the toxic and non-toxic isomers of BHC and, probably, there are differences in the distribution of the poison in the bodies of the susceptible and resistant strains. Resistant strains also convert a greater percentage of the absorbed dose in the earlier stages of storage.

By reversed-phase chromatography it has been established that the carbon tetrachloride extracts of flies treated with $\gamma$ - and $\delta$-BHC contain mainly unchanged BHC. No dehydrochlorination products have been detected in these extracts, and evidence that metabolism is accompanied by dechlorination was presented. The problem of the identification of the metabolic products is complex. In the metabolism of the four common isomers of BHC, watersoluble compounds are produced most readily from the $\alpha$ - and $\gamma$-isomers and much less readily from the $\delta$ - and $\beta$-forms. Two-dimensional chromatography has revealed eleven well-defined compounds from both $\gamma$ - and $\alpha$-BHC; these have not been identified, but there is evidence from paper electrophoresis that eight of the eleven compounds from these two stereoisomers are the same. It is unlikely that all the metabolites are trichlorobenzene derivatives, and it is possible that benzene derivatives (by dechlorinetion) are intermediate stages in the production of some of these metabolites. Comparative tests on a number of insect species have shown that even normal houseflies are exceptionally able to convert $\gamma$-BHC to excretable, water-soluble metabolites, and this factor may predispose this insect to the acquisition of insecticide resistance.

It is concluded that the reduced absorption and the increased amount of metabolism are not alone sufficient to explain the increased resistance. These two factors give at the most a sixfold difference in BHC content between normal and resistant flies, while resistance has increased by a factor of one hundred. However, it is possible that a combination of reduced absorption, increased metabolism and redistribution of the poison in the insect might provide the explanation.

The concluding paper in the afternoon was given by F. P. W. Winteringham on biochemical factors in the natural and acquired tolerance of insects to insecticides. It was pointed out that, when an insecticide is applied to an insect, it may be attacked by the tissue enzymes with the formation of metabolites which may be more or less toxic than the insecticide itself. An example where the metabolite is more toxic than the original chemical is parathion, which is a poor inhibitor of cholinesterase. Parathion is known to be oxidized by the insect in vivo to the oxygen analogue paraoxon, which is a potent inhibitor of cholinesterase. Similarly, it has been established that 'Schradan' becomes oxidized to a monophosphoramide oxide or a compound very much like it. The process occurring in these two examples might be referred to as toxication. The conversion of insecticides to harmless metabolites or detoxification has been demonstrated with DDT and BHC, particularly with insects resistant to these poisons. It has now also been established that the pyrethroids undergo detoxification in the adult housefly, and there is some evidence that the detoxification process is not one of simple hydrolysis.

The increased detoxification of poisons by resistant insects when compared with normal insects might be either a consequence or a cause of the insects' survival, there being evidence for and against either possibility. Extensive detoxification may occur in normal insect strains, and this must be a factor in their normal tolerance. Detoxification may be inhibited by the presence of certrin adjuvants applied simultaneously with the insecticide. A complete understanding of the DDT-resistance problem implies a better understanding of DDT action in viro. Recent work indicates that DDT may, in normal strains of insect, interfere with the oxidative formation of the vital tissue substance adenosine triphosphate. The interference was not observed in a resistant strain, and it may therefore be an additional biochemical factor in DDT-resistance mechanism.

When the evidence provided by all the papers given at the symposium is considered, it appears that results are steadily being accumulated on the changes of resistance that occur naturally and that are produced by the application of an insecticide. However, little progress has, as yet, been made in demonstrating the causes for the differences in resistance that are found, and this work is severely handicapped by lack of knowledge of the mode of action of insecticides.

\section{THE 74-IN. REFLECTOR OF THE COMMONWEALTH OBSERVATORY, MOUNT STROMLO, CANBERRA}

$\mathrm{E}^{\mathrm{A}}$ ARLY in 1947, the Commonwealth Astronomer, Prof. R. v. d. R. Woolley, supported by the then Astronomer Royal, Sir Harold Spencer Jones, and the board of visitors of the Commonwealth Observatory at Canberra, laid before the Minister for the Interior, the Hon. H. V. Johnson, proposals relating to the establishment of a large reflector at Mount Stromlo.

Among the arguments advanced for the placing of such a telescope at Mount Stromlo was the fact that telescopes of 72-in. aperture or more are to be found almost exclusively in the northern hemisphere. Even at the present time, with the Stromlo instrument operating, the situation is still unbalanced, for there are two such instruments in the south as against nine (including those under construction) in the north.

Cabinet approval was given for the construction of the instrument, and in 1948 an order for a 74-in. telescope complete with building and dome was 